\title{
Variational Approach for Solving the Ponds Seepage Problem
}

\author{
Fadhel S. Fadhel $^{*}$ and Isam H. Khayoon ${ }^{* *}$ \\ Department of Mathematics, College of Science, Al-Nahrain University, Baghdad-Iraq \\ Department of Mathematics, College of Education for pure Science, Ibn AL-Haitham, University, \\ Baghdad - Iraq \\ Corresponding Author: aaaaa_hhhhh2270@yahoo.com ${ }^{* *}$, dr_fadhel67@yahoo.com*
}

\begin{abstract}
In this paper, the function of the pond's seepage problem is derived using versional approach as especial case of generalized dam problem. In this problem the two dimensional cross section will produce problem with two free surfers which are evaluated as a part of the problem using simulating computer program.

[DOI: $\underline{10.22401 / \mathrm{JNUS} .21 .3 .17]}$
\end{abstract}

Keywords: Variational approach, free boundary value problem, ponds seepage problem, Magri's approach.

\section{Introduction}

The topic that is closely related to differential equations is the calculus of variation, which deals with the problem of maximizing or minimizing functional that is variable values which depend on a variable running through a set of functions, or on a finite number of such variables, and which are. Completely determined by a definite choice of these variable functions problems that consist of finding maxima or minima of a functional are called variational problem, [7].

The phrase "variational formulation" had been used recently in connection with generalized formulation of boundary -or initial value problems. In boundary value problems, sometimes it happens that a part of the boundary is unknown and must be determined as a part of the solution. This unknown boundary occurs in two cases, the first of which is called the moving boundary, which occurs mostly in heat-flow problems with phase changes and in certain diffusion processes. The second type is called, a free boundary which does not move but its position has to be determined as a part of the solution of a steady-state problem, [3].

The main objective of this article is to deal in general with seepage through porous media, which is an important source of free boundary problems, for example the seepage through earth dams, seepage out of open channels such as rivers, canals, ponds and irrigation system, .etc. [1].

Historically, as a literature survey the essential features of variational methods goes back approximately for more than two centuries when the first notions of the subject for variational of calculus began to be formulated. Actually, the most primitive ideas of variational theory had been presented first in Ariistothes writings on virtual velocities in 300 B.C., then they were reviewed by Galileo in the sixteenth century. Later, they were formulated into a principle of virtual work by John Bernolli in 1717. The first step toward developing a general method for solving variational problems was given by Euler in 1732, through presenting "a general solution of the isoperimetric problem". It was in this work and subsequent writing of Euler that variational concepts found a welcome and permanent home in mechanics, $[4,6]$.

A more solid mathematical basis for variational theory began to be developed in the eighteenth and early nineteenth century. Necessary conditions for the existence of "minimizing curves" of a certain functional were studied during this period and we found among contributors of that area the familiar names of Legendre, Jacobi and Weirstrass, [5].

Legendre gave criteria for distinguishing between maxima and minima in 1786 without considering criteria for existence. Jacobi gave sufficient conditions for existence of an extrema of a functional in 1837, [9]. The main problem in calculus of variation is to find the maximum or minimum values of a given functional $\mathrm{J}(\mathrm{y})$, this necessary condition is called the Euler-Lagrange equation. This problem is called, for simplicity, the direct problem of calculus of variation [4]. 
Functional are variable values which depend on a variable function running through a set of functions or on a finite number of such variables, and which are completely determined by a definite choice of these variable functions, [6].

At the end of the nineteenth century and in the early years of the twentieth century, we found prominent contributions to the subject of variational ideas, particularly, in the area of problems, were Ritz, Galerkin and Hellinger are the pioneers.

Variational concepts now play a fundamental role in applied mathematics. As an example, the solution of any ordinary problems, such as partial differential equations, ordinary differential equations, integral equations, etc. is equivalent to the minimization of the functional $\mathrm{J}$ that corresponds to this ordinary equation, [10].

As it is well known, the initiation of the study of variational principles should be attributed to Euler and Lagrange and in a broader setting, to Poisson, Cauchy and Hamilton.

In recent time, the development of a unified theory for linear problems is given, where Hussain E. A. in 1987 studied the solution of the boundary value problems using variational approach, [8]. Also, Mahlol in 1993, studied the solution of the direct and inverse problems of eigenvalue problems, then studying its application for localizing the size of Brain tumors, [11]. In addition, among other studies concerning the direct and inverse problems with application, the study of Ali J. A. in 1994 for the mathematical inverse problem of acoustic wave scattering, [2]. Jabbar in 2001, consider the solution of the two-dimensional moving value problems of Hele-Show problem, [9]. Finally, Al-Ani in 2001 consider the study of the twodimensional inverse problem of the seepage in a simple rectangular dam, [1].

In this paper we will solve the seepage through a pond which is considered as an application to the generalized dam problem, this had been dam by using variational approach, as well as, evaluate its numerical solution. Numerical simulation is carried using computer program written in MATLAB 2016a for this purpose.

\section{Preliminaries}

In this section, some necessary and basic most fundamental concepts related to this subject of calculus of variation which are useful for understudy this paper are presented for completeness purpose the corner stone of the variational approach is the formulation of the functional related to the problem under consideration using Magri's approach, [12]. This approach needs some basic concepts, which are given in the next definitions.

\section{Definition (1), [14]:}

Let $\mathrm{U}$ and $\mathrm{V}$ be two normed linear spaces, a bilinear form defined on $\mathrm{U}$ and $\mathrm{V}$ is a functional $\mathrm{L}: \mathrm{U} \times \mathrm{V} \longrightarrow \square$, which is linear in both $\mathrm{U}$ and $\mathrm{V}$, where $\mathrm{u}$ and $\mathrm{v}$ are elements of $\mathrm{U}$ and $\mathrm{V}$ respectively, and the following properties are fulfilled:

$$
\begin{aligned}
& \text { 1. } \mathrm{L}\left(\alpha_{1} \mathrm{u}+\alpha_{2} \mathrm{w}, \mathrm{v}\right)=\alpha_{1} \mathrm{~L}(\mathrm{u}, \mathrm{v})+\alpha_{2} \mathrm{~L}(\mathrm{w}, \mathrm{v}) \text {, } \\
& \alpha_{1}, \alpha_{2} \in \square, \mathrm{u}, \mathrm{w} \in \mathrm{U}, \mathrm{v} \in \mathrm{V} \text {. } \\
& \text { 2. } \mathrm{L}\left(\mathrm{u}, \beta_{1} \mathrm{v}+\beta_{2} \mathrm{w}\right)=\beta_{1} \mathrm{~L}(\mathrm{u}, \mathrm{v})+\beta_{2} \mathrm{~L}(\mathrm{v}, \mathrm{w}) \text {, } \\
& \beta_{1}, \beta_{2} \in \square, \mathrm{u} \in \mathrm{U}, \mathrm{v}, \mathrm{w} \in \mathrm{V} \text {. }
\end{aligned}
$$

this functional is usually denoted by the symbol $\langle\mathrm{u}, \mathrm{v}\rangle$.

\section{Definition (2), [14]:}

Let $\langle\mathrm{u}, \mathrm{v}\rangle$ be a bilinear form then:

1. $\langle\mathrm{u}, \mathrm{v}\rangle$ is said onto be symmetric if $\langle\mathrm{u}, \mathrm{v}\rangle=\langle\mathrm{v}, \mathrm{u}\rangle$ for all $\langle\mathrm{u}, \mathrm{v}\rangle \in \mathrm{U} \times \mathrm{V}$

2. The bilinear form $\langle\mathrm{u}, \mathrm{v}\rangle$ is non-degenerate on $\mathrm{U}$ and $\mathrm{V}$ if

$$
\begin{aligned}
& \text { i. }\langle\mathrm{u}, \overline{\mathrm{v}}\rangle=0 \rightarrow \overline{\mathrm{v}}=0, \forall \mathrm{u} \in \mathrm{U} \\
& \text { ii. }\langle\overline{\mathrm{u}}, \mathrm{v}\rangle=0 \rightarrow \overline{\mathrm{u}}=0, \forall \mathrm{v} \in \mathrm{V}
\end{aligned}
$$

Among the following most usual examples of non-degenerate bilinear forms:

$$
\langle\mathrm{u}, \mathrm{v}\rangle=\int_{0}^{\tau} \mathrm{u}(\mathrm{t}) \mathrm{v}(\mathrm{t}) \mathrm{dt}
$$

where $\mathrm{u}, \mathrm{v}: \mathrm{C}[0, \tau] \longrightarrow \square, \tau>0$.

$$
\langle\mathrm{u}, \mathrm{v}\rangle=\int_{0}^{\tau} \sum_{\mathrm{n}} \mathrm{u}_{\mathrm{n}}(\mathrm{t}) \mathrm{v}_{\mathrm{n}}(\mathrm{t}) \mathrm{dt}
$$

where $\mathrm{u}, \mathrm{v}: \mathrm{C}[0, \tau] \longrightarrow \square^{\mathrm{n}}, \tau>0$. 


$$
\langle\mathrm{u}, \mathrm{v}\rangle=\int_{0}^{\tau} \mathrm{u}(\mathrm{t}) \mathrm{v}(\tau-\mathrm{t}) \mathrm{dt}
$$

where $\mathrm{u}, \mathrm{v}: \mathrm{C}[0, \tau] \longrightarrow \square, \tau>0$.

$$
\langle\mathrm{u}, \mathrm{v}\rangle=\int_{0}^{\tau} \mathrm{u}(\mathrm{t})\left[\int_{\mathrm{s}=0}^{\mathrm{s}=\tau-\mathrm{t}} \mathrm{K}(\mathrm{t}, \mathrm{s}) \mathrm{v}(\mathrm{s}) \mathrm{ds}\right] \mathrm{dt}
$$

where $\mathrm{u}, \mathrm{v}: \mathrm{C}[0, \tau] \longrightarrow \square, \tau>0$.

For problem modeled using PDE's, the must usual used non-degenerate bilinear form is given by:

$$
\langle\mathrm{u}, \mathrm{v}\rangle=\int_{0}^{\tau} \int_{0}^{\tau} \mathrm{u}(\mathrm{x}, \mathrm{t}) \mathrm{v}(\mathrm{x}, \mathrm{t}) \mathrm{dtdx}
$$

Where u, v: C ([0, t ] $\times[0, \mathrm{t}]) \rightarrow \square, \mathrm{t}>0$.

\section{Definition (3), [14]:}

A given linear operator $\mathrm{L}: \mathrm{D}(\mathrm{L}) \rightarrow \mathrm{R}(\mathrm{L})$ is called symmetric with respect to the chosen bilinear form $\langle\mathrm{u}, \mathrm{v}\rangle$ if it satisfies:

$$
\langle\mathrm{Lu}, \mathrm{v}\rangle=\langle\mathrm{Lv}, \mathrm{u}\rangle
$$

Finally, we end this section with the mathematical theorem which is called in some literation's Magri's_approach that is used to evaluate the functional related to the initialboundary value problem under consideration and have to be so lead.

\section{Theorem (1), [14]:}

There is a variational problem $\left.\mathrm{J}(\mathrm{u})=\frac{1}{2}<\mathrm{Lu}, \mathrm{u}\right\rangle-<\mathrm{f}, \mathrm{u}>$ corresponding to initial-boundary value problems $\mathrm{Lu}=\mathrm{f}$, if and only if the operator $\mathrm{L}$ is symmetric relative to the chosen bilinear form which is nondegenerate.

It is remarkable that, the elementary concepts of calculus of variation are not presented here and are considered to be known to the readers (for more derails see $[6,13,10]$.

\section{Mathematical Formulation of the Problem}

The mathematical formulation and modeling the pond seepage problem must pass through the physical derivation of the problem, which will not presented here using by Darcy's low for deriving the continuity equation and velocity potential function of the problem, [15].

The mathematical modeling of the problem is formulated as a free boundary value problem and have the governing equation with initial and boundary condition of the pond seepage problem, which will has the form (see Fig.(1))

$$
\Phi_{\mathrm{xx}}+\Phi_{\mathrm{yy}}=0 \quad,(\mathrm{x}, \mathrm{y}) \in \Omega \mathrm{R} \text { or } \Omega \mathrm{L}
$$

with initial and boundary condition for the right dam side

$$
\left.\begin{array}{lrr}
\operatorname{} R_{\mathrm{y}}(\mathrm{x}, 0)=0 & , & 0 \leq \mathrm{x} \leq \mathrm{LR} \\
\Phi \mathrm{R}\left(\mathrm{x}, \mathrm{ER}_{1}(\mathrm{x})\right)=\mathrm{HR}_{0} & , & 0 \leq \mathrm{x} \leq \mathrm{xR}_{0} \\
\Phi \mathrm{R}\left(\mathrm{x}, \mathrm{ER}_{2}(\mathrm{x})\right)=\mathrm{HR}_{1} & , & \mathrm{xR}_{2} \leq \mathrm{x} \leq \mathrm{LR} \\
\Phi \mathrm{R}(\mathrm{x}, \mathrm{HR}(\mathrm{x}))=\mathrm{HR}(\mathrm{x}) & , & \mathrm{xR}_{0} \leq \mathrm{x} \leq \mathrm{xR}_{1} \\
\Phi \mathrm{R}\left(\mathrm{x}, \mathrm{ER}_{2}(\mathrm{x})\right)=\mathrm{ER}_{2}(\mathrm{x}) & , & \mathrm{xR}_{1} \leq \mathrm{x} \leq \mathrm{xR}_{2}
\end{array}\right\}
$$

Also, the initial and boundary conditions for the left dam side one given by

$$
\left.\begin{array}{lrr}
\Phi \mathrm{L}_{\mathrm{y}}(\mathrm{x}, 0)=0 & , & 0 \leq \mathrm{x} \leq \mathrm{LL} \\
\Phi \mathrm{L}\left(\mathrm{x}, \mathrm{EL}_{1}(\mathrm{x})\right)=\mathrm{HL}_{0} & , & 0 \leq \mathrm{x} \leq \mathrm{xL}_{0} \\
\Phi \mathrm{L}\left(\mathrm{x}, \mathrm{EL}_{2}(\mathrm{x})\right)=\mathrm{HL}_{1} & , & \mathrm{xL}_{2} \leq \mathrm{x} \leq \mathrm{LL} \\
\Phi \mathrm{L}(\mathrm{x}, \mathrm{HL}(\mathrm{x}))=\mathrm{HL}(\mathrm{x}) & , & \mathrm{xL}_{0} \leq \mathrm{x} \leq \mathrm{xL}_{1} \\
\Phi \mathrm{L}\left(\mathrm{x}, \mathrm{EL}_{2}(\mathrm{x})\right)=\mathrm{EL}_{2}(\mathrm{x}) & , & \mathrm{xL}_{1} \leq \mathrm{x} \leq \mathrm{xL}_{2}
\end{array}\right\}
$$

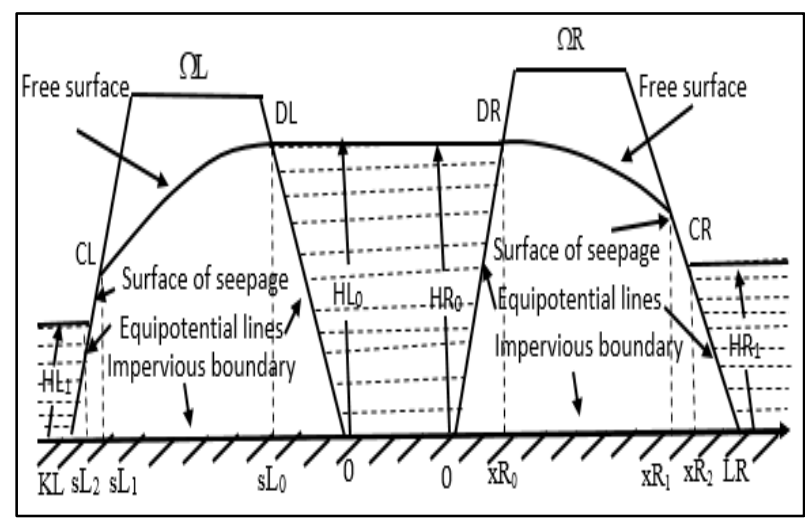

Fig.(1) The free surface is assumed to seepage.

\section{Variational Formulation of the Problem}

To solve pond seepage problem, we have to solve equation with its related boundary and 
initial conditions, as well as the evaluation of the free surface as a part of solution of the problem. We turn in this section to the variational formulation of the problem. The related functional derived using Magri's approach is given by:

$$
\mathrm{J}(\Phi)=\iint_{\Omega}\left[\Phi_{\mathrm{x}}^{2}+\Phi_{\mathrm{y}}^{2}\right] \mathrm{dxdy}
$$

Where $\Omega=\Omega_{1} U \Omega_{2}$, and $\Omega_{1}$ is the right sided seepage region which is decomposed for computation purpose into the following sub regions

$\mathrm{RR}_{1}=\left\{(\mathrm{x}, \mathrm{y}): 0 \leq \mathrm{x} \leq \mathrm{xR} \mathrm{R}_{0}, 0 \leq \mathrm{y} \leq \mathrm{ER}_{1}(\mathrm{x})\right\}$

$\mathrm{RR}_{2}=\left\{(\mathrm{x}, \mathrm{y}): \mathrm{xR}_{0} \leq \mathrm{x} \leq \mathrm{xR} \mathrm{R}_{1}, 0 \leq \mathrm{y} \leq \mathrm{HR}(\mathrm{x})\right\}$

$\mathrm{RR}_{3}=\left\{(\mathrm{x}, \mathrm{y}): \mathrm{xR}_{1} \leq \mathrm{x} \leq \mathrm{xR} \mathrm{R}_{2}, 0 \leq \mathrm{y} \leq \mathrm{ER}_{2}(\mathrm{x})\right\}$

$\mathrm{RR}_{4}=\left\{(\mathrm{x}, \mathrm{y}): \mathrm{xR} \mathrm{R}_{2} \leq \mathrm{x} \leq \mathrm{LR}, 0 \leq \mathrm{y} \leq \mathrm{ER}_{2}(\mathrm{x})\right\}$

while $\Omega_{2}$ is the left sided seepage region and also for computation purpose is decomposed into the following sub regions

$$
\begin{aligned}
& \mathrm{RL}_{1}=\left\{(\mathrm{x}, \mathrm{y}): 0 \leq \mathrm{x} \leq \mathrm{sL}_{0}, 0 \leq \mathrm{y} \leq \mathrm{ML}_{1}(\mathrm{x})\right\} \\
& \mathrm{RL}_{2}=\left\{(\mathrm{x}, \mathrm{y}): \mathrm{sL}_{0} \leq \mathrm{x} \leq \mathrm{sL}_{1}, 0 \leq \mathrm{y} \leq \mathrm{GL}(\mathrm{x})\right\} \\
& \mathrm{RL}_{3}=\left\{(\mathrm{x}, \mathrm{y}): \mathrm{sL}_{1} \leq \mathrm{x} \leq \mathrm{sL}_{2}, 0 \leq \mathrm{y} \leq \mathrm{ML}_{2}(\mathrm{x})\right\} \\
& \mathrm{RL}_{4}=\left\{(\mathrm{x}, \mathrm{y}): \mathrm{sL}_{2} \leq \mathrm{x} \leq \mathrm{KL}, 0 \leq \mathrm{y} \leq \mathrm{ML}_{2}(\mathrm{x})\right\}
\end{aligned}
$$

Hence, the functional $\mathbf{J}$ may be rewritten for $\Omega_{1}$ into form:

$$
\begin{aligned}
\mathrm{J}(\Phi)= & \int_{0}^{\mathrm{xR} 0} \int_{0}^{\mathrm{ER} 1(\mathrm{x})}\left[\Phi_{\mathrm{x}}^{2}+\Phi_{\mathrm{y}}^{2}\right] \mathrm{dydx} \\
& +\int_{\mathrm{xR} 0}^{\mathrm{xR} 1} \int_{0}^{\mathrm{HR}(\mathrm{x})}\left[\Phi_{\mathrm{x}}^{2}+\Phi_{\mathrm{y}}^{2}\right] \mathrm{dydx} \\
& +\int_{\mathrm{xR} 1}^{\mathrm{xR} 2} \int_{0}^{\mathrm{ER} 2(\mathrm{x})}\left[\Phi_{\mathrm{x}}^{2}+\Phi_{\mathrm{y}}^{2}\right] \mathrm{dydx} \\
& +\int_{\mathrm{xR} 2}^{\mathrm{LR}} \int_{0}^{\mathrm{ER} 2(\mathrm{x})}\left[\Phi_{\mathrm{x}}^{2}+\Phi_{\mathrm{y}}^{2}\right] \mathrm{dydx}
\end{aligned}
$$

and $\Omega_{2}$ respectively in the following form:

$$
\begin{aligned}
J(\Phi)= & \int_{0}^{\operatorname{sL} 0} \int_{0}^{\operatorname{ML} 1(\mathrm{x})}\left[\Phi_{\mathrm{x}}^{2}+\Phi_{\mathrm{y}}^{2}\right] \mathrm{dydx} \\
& +\int_{\mathrm{sL} 0}^{\mathrm{sL} 1} \int_{0}^{\mathrm{GL}(\mathrm{x})}\left[\Phi_{\mathrm{x}}^{2}+\Phi_{\mathrm{y}}^{2}\right] \mathrm{dydx}
\end{aligned}
$$

$$
\begin{aligned}
& +\int_{\mathrm{sL} 1}^{\mathrm{sL} 2} \int_{0}^{\operatorname{ML} 2(\mathrm{x})}\left[\Phi_{\mathrm{x}}^{2}+\Phi_{\mathrm{y}}^{2}\right] \mathrm{dydx} \\
& +\int_{\mathrm{sL} 2}^{\operatorname{KL}} \int_{0}^{\operatorname{ML} 2(\mathrm{x})}\left[\Phi_{\mathrm{x}}^{2}+\Phi_{\mathrm{y}}^{2}\right] \mathrm{dydx}
\end{aligned}
$$

\section{Numerical Simulation of the Problem}

In order to solve the problem of this paper, numerical simulation is carried using computer program written in MATLAB 2016a for this purpose, suppose for $\Omega_{1}$ the following parameter are considered $\mathrm{x}_{0}=0.5, \mathrm{x}_{1}=1, \mathrm{x}_{2}=1.5$, $\mathrm{L}=2, \mathrm{H}_{0}=1.0, \mathrm{H}_{1}=0.5$. Also, the free surface is assumed to be

$$
\mathrm{H}(\mathrm{x})=\mathrm{b}_{0}+\mathrm{b}_{1}\left(\mathrm{x}-\mathrm{x}_{0}\right)+\mathrm{b}_{2}\left(\mathrm{x}-\mathrm{x}_{0}\right)^{2}+\mathrm{b}_{3}\left(\mathrm{x}-\mathrm{x}_{0}\right)^{3}
$$

applying the boundary conditions, the formula becomes as follows:

$$
\mathrm{H}(\mathrm{x})=\mathrm{H}_{0}-\frac{1}{\mathrm{M}_{0}}\left(\mathrm{x}-\mathrm{x}_{0}\right)+\mathrm{B}\left(\mathrm{x}-\mathrm{x}_{0}\right)^{2}+\mathrm{b}_{3}\left(\mathrm{x}-\mathrm{x}_{0}\right)^{3}
$$

So, $\mathrm{b}_{0}=\mathrm{H}_{0}, \mathrm{~b}_{1}=-\frac{1}{\mathrm{M}_{0}}, \mathrm{~b}_{2}=\mathrm{B}$,

OD: the height of the dam which passes through the origin $(0,0)$. If the slope of the rectum is $\mathrm{M}_{0}$, the $\mathrm{M}_{0}$ is given with the following relationship:

(See Fig. (2))

$$
\mathrm{M}_{0}=\frac{\mathrm{DN}}{\mathrm{ON}}
$$

and

$$
\text { if } \mathrm{ON}=\mathrm{x}_{0} \text {, }
$$

then

$$
\mathrm{M}_{0}=\frac{\mathrm{H}_{0}}{\mathrm{x}_{0}} \quad, \mathrm{x}_{0} \neq 0
$$

$\mathrm{AB}$ : the dam reservoir side, which passes through the point $(\mathrm{L}, 0)$, where $\mathrm{L}$ is the length of the base of the dam. If the slope of this rectangle $\mathrm{M}_{1}, \mathrm{M}_{1}$ may be given as follows relationship: (see Fig. (2))

$$
\begin{aligned}
& \mathrm{AM}=\mathrm{L}-\mathrm{x}_{2} \\
& \mathrm{OM}=\mathrm{x}_{2} \\
& \mathrm{M}_{1}=\frac{\mathrm{MB}}{\mathrm{AM}}, \mathrm{AM} \neq 0
\end{aligned}
$$


therefore:

$\mathrm{M}_{1}=\frac{\mathrm{H}_{1}}{\mathrm{~L}-\mathrm{x}_{2}}, \mathrm{~L} \neq \mathrm{x}_{2}$

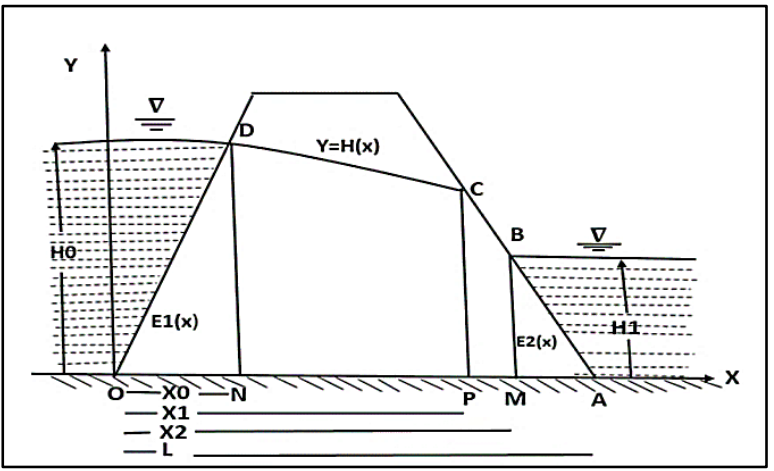

Fig.(2): Cross sectional through twodimensional dam.

$\mathrm{b}_{3}=\frac{\mathrm{M}_{1}+\frac{1}{\mathrm{M}_{0}}+2 \mathrm{~B}\left(\mathrm{x}_{1}-\mathrm{x}_{0}\right)}{3\left(\mathrm{x}_{1}-\mathrm{x}_{0}\right)^{2}}, \mathrm{x}_{1} \neq \mathrm{x}_{0}$

The approximation of the function $\Phi$ using Ritz method over the four sub reigns $R_{1}$, $R_{2}, R_{3}$ and $R_{4}$, respectfully are:

$\Phi_{1}=\mathrm{y}^{2}\left(\mathrm{y}-\mathrm{E}_{1}(\mathrm{x})\right)(\mathrm{A}+\mathrm{CX}+\mathrm{DY})+\mathrm{H}_{0} \quad, 0 \leq \mathrm{x} \leq \mathrm{x}_{0}$

$\Phi_{2}=\mathrm{y}^{2}(\mathrm{y}-\mathrm{H}(\mathrm{x}))(\mathrm{A}+\mathrm{CX}+\mathrm{DY})+\mathrm{H}(\mathrm{x}), \mathrm{x}_{0} \leq \mathrm{x} \leq \mathrm{x}_{1}$

$\Phi_{3}=\mathrm{y}^{2}\left(\mathrm{y}-\mathrm{E}_{2}(\mathrm{x})\right)(\mathrm{A}+\mathrm{CX}+\mathrm{DY})+\mathrm{E}_{1}(\mathrm{x}), \mathrm{x}_{1} \leq \mathrm{x} \leq \mathrm{x}_{2}$

$\Phi_{4}=\mathrm{y}^{2}\left(\mathrm{y}-\mathrm{E}_{2}(\mathrm{x})\right)(\mathrm{A}+\mathrm{CX}+\mathrm{DY})+\mathrm{H}_{1}, \mathrm{x}_{2} \leq \mathrm{x} \leq \mathrm{L}$

In order to find the coefficients $\mathrm{A}, \mathrm{B}, \mathrm{C}$ and $\mathrm{D}$ which minimized the functional (5), we evaluate the partial derivations of $\mathrm{J}$ with respect to those constants and everting to zero will leads to a liner system ,i.e., if

$\Phi_{1 x}=\mathrm{y}^{2}\left(\mathrm{y}-\mathrm{E}_{1}(\mathrm{x})\right) \mathrm{C}+\mathrm{y}^{2}\left(-\mathrm{E}_{1}^{\prime}(\mathrm{x})\right)(\mathrm{A}+\mathrm{CX}+\mathrm{DY}) \quad, \quad 0 \leq \mathrm{x} \leq \mathrm{x}_{0}$

$\Phi_{2 x}=y^{2}(y-H(x)) C+y^{2}\left(-H^{\prime}(x)\right)(A+C X+D Y)+H^{\prime}(x), x_{0} \leq x \leq x_{2}$

$\Phi_{3 x}=\mathrm{y}^{2}\left(\mathrm{y}-\mathrm{E}_{2}(\mathrm{x})\right) \mathrm{C}+\mathrm{y}^{2}\left(-\mathrm{H}^{\prime}(\mathrm{x})\right)(\mathrm{A}+\mathrm{CX}+\mathrm{DY})-\mathrm{M}, \mathrm{x}_{1} \leq \mathrm{x} \leq \mathrm{x}_{2}$

$\Phi_{4 x}=y^{2}\left(y-E_{2}(x)\right) C+Y^{2}\left(-E_{2}^{\prime}(x)\right)\left(A+C X+D Y \quad, \quad x_{2} \leq x \leq L\right.$

and

$\left.\left.\Phi_{1 y}=3 y^{2}-2 y E_{1}(x)\right)(A+C X+D Y)+y^{3}-y^{2} E_{1}(x)\right) D, 0 \leq x \leq x_{0}$

$\left.\Phi_{2 y}=3 \mathrm{y}^{2}-2 \mathrm{yH}(\mathrm{x})\right)(\mathrm{A}+\mathrm{CX}+\mathrm{DY})+\left(\mathrm{y}^{3}-\mathrm{y}^{2} \mathrm{H}(\mathrm{x})\right) \mathrm{D}, \mathrm{x}_{0} \leq \mathrm{x} \leq \mathrm{x}_{1}$

$\Phi_{3 y}=3 \mathrm{y}^{2}-2 \mathrm{yH}(\mathrm{x})(\mathrm{A}+\mathrm{CX}+\mathrm{DY})+\left(\mathrm{y}^{3}-\mathrm{y}^{2} \mathrm{E}_{2}(\mathrm{x})\right) \mathrm{D}, \mathrm{x}_{1} \leq \mathrm{x} \leq \mathrm{x}_{2}$

$\left.\Phi_{4 y}=3 \mathrm{y}^{2}-2 \mathrm{yE}_{2}(\mathrm{x})\right)(\mathrm{A}+\mathrm{CX}+\mathrm{DY})+\left(\mathrm{y}^{3}-\mathrm{y}^{2} \mathrm{E}_{2}(\mathrm{x})\right) \mathrm{D}, \mathrm{x}_{2} \leq \mathrm{x} \leq \mathrm{L}$
Then

$$
\begin{aligned}
& \frac{\partial \mathrm{I}}{\partial \mathrm{A}}=\int_{0}^{\mathrm{x}_{0}} \int_{0}^{\mathrm{E}_{1}(\mathrm{x})}\left[2 \Phi_{1 \mathrm{x}}\left(\mathrm{y}^{2}\left(-\mathrm{E}_{1}^{\prime}(\mathrm{x})\right)+2 \Phi_{1 \mathrm{y}}\left(3 \mathrm{y}^{2}-2 \mathrm{y} \mathrm{E}_{1}(\mathrm{x})\right)\right] \mathrm{dydx}\right. \\
& +\int_{x_{0}}^{x_{1}} \int_{0}^{H(x)}\left[2 \Phi_{2 x}\left(y^{2}\left(-H^{\prime}(x)\right)+2 \Phi_{2 y}\left(3 y^{2}-2 y H(x)\right)\right] d y d x\right. \\
& +\int_{x_{1}}^{x_{1} E_{0}} \int_{0}^{E_{2}(x)}\left[2 \Phi_{3 x}\left(y^{2}\left(-E_{2}^{\prime}(x)\right)+2 \Phi_{3 y}\left(3 y^{2}-2 y E_{2}(x)\right)\right] d y d x\right. \\
& +\int_{x_{2}}^{L} \int_{0}^{\mathrm{E}_{2}(\mathrm{x})}\left[2 \Phi_{4 \mathrm{x}}\left(\mathrm{y}^{2}\left(-\mathrm{E}_{2}^{\prime}(\mathrm{x})\right)+2 \Phi_{4 \mathrm{y}}\left(3 \mathrm{y}^{2}-2 \mathrm{y} \mathrm{E}_{2}(\mathrm{x})\right)\right] \mathrm{dydx}\right.
\end{aligned}
$$

and

$$
\begin{aligned}
\frac{\partial \mathrm{I}}{\partial \mathrm{B}}= & \int_{\mathrm{x}_{0}}^{\mathrm{x} 1}\left[\left.\left(\left(\mathrm{x}-\mathrm{x}_{0}\right)^{2}+\frac{2\left(\mathrm{x}-\mathrm{x}_{0}\right)^{3}}{3\left(\mathrm{x}_{1}-\mathrm{x}_{0}\right)}\right)\left(\Phi_{2 \mathrm{x}}^{2}+\Phi_{2 \mathrm{y}}^{2}\right)\right|_{\mathrm{y}=\mathrm{H}(\mathrm{x})}\right] \mathrm{dx} \\
& +\int_{\mathrm{x}_{0}}^{\mathrm{x} 1} \int_{0}^{\mathrm{H}(\mathrm{x})} 2 \Phi_{2 \mathrm{x}}\left(\mathrm{y}^{2}\left(-\left(\mathrm{x}-\mathrm{x}_{0}\right)^{2}-\frac{2\left(\mathrm{x}-\mathrm{x}_{0}\right)^{3}}{3\left(\mathrm{x}_{1}-\mathrm{x}_{0}\right)}\right) \mathrm{C}\right. \\
& +\mathrm{y}^{2}\left(-2\left(\mathrm{x}-\mathrm{x}_{0}\right)-\frac{2\left(\mathrm{x}-\mathrm{x}_{0}\right)^{2}}{\mathrm{x}_{1}-\mathrm{x}_{0}}\right)(\mathrm{A}+\mathrm{Cx}+\mathrm{Dy}) \\
& +2 \Phi_{2 \mathrm{y}}\left(-2 \mathrm{y}\left(\left(\mathrm{x}-\mathrm{x}_{0}\right)^{2}+\frac{2\left(\mathrm{x}-\mathrm{x}_{0}\right)^{3}}{3\left(\mathrm{x}_{1}-\mathrm{x}_{0}\right)}\right)(\mathrm{A}+\mathrm{Cx}+\mathrm{Dy})\right. \\
& \left.\left.-\mathrm{y}^{2} \mathrm{D}\left(\mathrm{x}-\mathrm{x}_{0}\right)+\frac{2\left(\mathrm{x}-\mathrm{x}_{0}\right)^{3}}{3\left(\mathrm{x}_{1}-\mathrm{x}_{0}\right)}\right)\right) \mathrm{dydx}
\end{aligned}
$$

and 


$$
\begin{aligned}
\frac{\partial I}{\partial C}= & \int_{0}^{x_{0}} \int_{0}^{E_{1}(x)}\left[2 \Phi_{1 x}\left(\left(y^{2}\left(y-E_{1}(x)\right)+y^{2}\left(-E_{1}(x)\right) x\right)+2 \Phi_{1 y}\left(\left(3 y^{2}-2 y E_{1}(x)\right) x\right)\right] d y d x\right. \\
& +\int_{x_{0}}^{x_{1}} \int_{0}^{H(x)}\left[2 \Phi _ { 2 x } \left(\left(y^{2}(y-H(x))+y^{2}\left(-H^{\prime}(x) x\right)+2 \Phi_{2 y}\left(3 y^{2}-2 y H(x)\right) x\right] d y d x\right.\right. \\
& +\int_{x_{1}}^{x_{2}} \int_{0}^{E_{2}(x)}\left[2 \Phi_{3 x}\left(y^{2}\left(y-E_{2}(x)\right)+y^{2}\left(-E_{2}^{\prime}(x) x\right)+2 \Phi_{3 y}\left(3 y^{2}-2 y E_{2}(x)\right) x\right] d y d x\right. \\
& +\int_{x_{2}}^{L_{2}} \int_{0}^{E_{2}(x)}\left[2 \Phi_{4 x}\left(y^{2}\left(y-E_{2}(x)\right)+y^{2}\left(-E_{2}^{\prime}(x) x\right)+2 \Phi_{4 y}\left(3 y^{2}-2 y E_{2}(x)\right) x\right] d y d x\right.
\end{aligned}
$$

and

$$
\begin{aligned}
\frac{\partial \mathrm{I}}{\partial \mathrm{D}}= & \int_{0}^{\mathrm{x}_{0}} \int_{0}^{\mathrm{E}_{1}(\mathrm{x})}\left[2 \Phi_{1 \mathrm{x}}\left(\mathrm{y}^{3}\left(-\mathrm{E}_{1}^{\prime}(\mathrm{x})\right)+2 \Phi_{1 \mathrm{y}}\left(\left(3 \mathrm{y}^{2}-2 \mathrm{y}^{2} \mathrm{E}_{1}(\mathrm{x})\right) \mathrm{y}\right)+\left(\mathrm{y}^{3}-\mathrm{y}^{2} \mathrm{E}_{1}(\mathrm{x})\right)\right)\right] \mathrm{dydx} \\
& +\int_{\mathrm{x}_{0}}^{\mathrm{x}_{1}} \int_{0}^{\mathrm{H}(\mathrm{x})}\left[2 \Phi_{2 \mathrm{x}}\left(\left(\mathrm{y}^{3}\left(-\mathrm{H}^{\prime}(\mathrm{x})\right)+2 \Phi_{2 \mathrm{y}}\left(3 \mathrm{y}^{3}-2 \mathrm{y}^{2} \mathrm{H}(\mathrm{x})\right) \mathrm{y}+\mathrm{y}^{3}-\mathrm{y}^{2} \mathrm{H}(\mathrm{x})\right)\right] \mathrm{dydx}\right. \\
& +\int_{\mathrm{x}_{1}}^{\mathrm{x}_{2}} \int_{0}^{\mathrm{E}_{2}(\mathrm{x})}\left[2 \Phi_{3 \mathrm{x}}\left(\mathrm{y}^{3}\left(-\mathrm{E}_{2}^{\prime}(\mathrm{x})\right)+2 \Phi_{3 \mathrm{y}}\left(3 \mathrm{y}^{3}-2 \mathrm{y}^{2} \mathrm{E}_{2}(\mathrm{x})\right) \mathrm{y}+\mathrm{y}^{3}-\mathrm{y}^{2} \mathrm{E}_{2}(\mathrm{x})\right)\right] \mathrm{dydx} \\
& +\int_{\mathrm{x}_{2}}^{\mathrm{L}_{2}} \int_{0}^{\mathrm{E}_{2}(\mathrm{x})}\left[2 \Phi_{4 \mathrm{x}}\left(\mathrm{y}^{3}\left(-\mathrm{E}_{2}^{\prime}(\mathrm{x})\right)+2 \Phi_{4 \mathrm{x}}\left(3 \mathrm{y}^{3}-\mathrm{y}^{2} \mathrm{E}_{2}(\mathrm{x})\right) \mathrm{y}+\mathrm{y}^{3}-\mathrm{y}^{2} \mathrm{E}_{2}(\mathrm{x})\right)\right] \mathrm{dydx}
\end{aligned}
$$

and upper carry the computer program we get the following results:

$\mathrm{H}_{0}=1.00, \mathrm{H}_{1}=0.5, \mathrm{x}_{0}=0.5, \mathrm{x}_{1}=1, \mathrm{x}_{2}=1.5, \mathrm{~L}=2$

$\mathrm{H}(\mathrm{x})=\mathrm{b}_{0}+\mathrm{b}_{1}\left(\mathrm{x}-\mathrm{x}_{0}\right)+\mathrm{b}_{2}\left(\mathrm{x}-\mathrm{x}_{0}\right)^{2}+\mathrm{b}_{3}\left(\mathrm{x}-\mathrm{x}_{0}\right)^{3}$

Then:

$\mathrm{A}=-0.0706, \mathrm{~B}=-0.0037, \mathrm{C}=-0.0677$,

$\mathrm{D}=-0.0403$

and

$\mathrm{b}_{0}=1.0000, \mathrm{~b}_{1}=0.5000, \mathrm{~b}_{2}=-1.0000$,

$b_{3}=-0.6667$

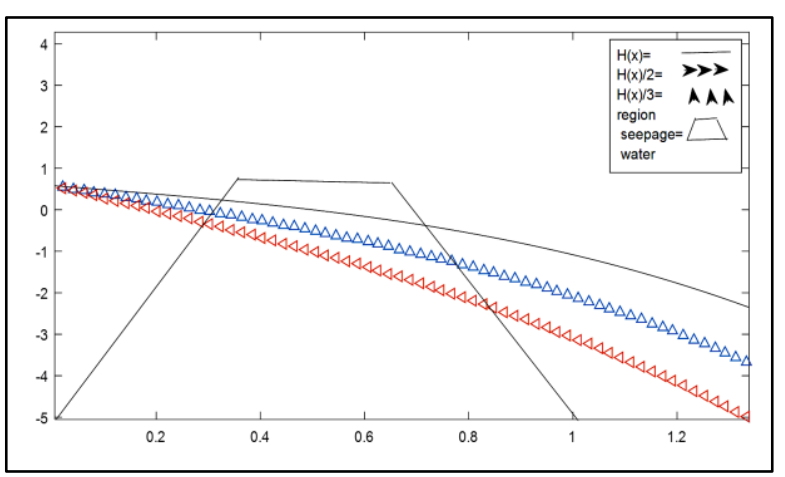

Fig. (3): Approximate free surface of the twodimensional simple.

Similarly, we carry out the simulation for the left dam region $\Omega_{2}$ with the free surface is assumed to be.

Using MATLAB R2016a, the following results have emerged when:

$$
\begin{aligned}
& \mathrm{H}_{0}=1.00, \mathrm{H}_{1}=0.5, \mathrm{~s}_{0}=-0.5, \mathrm{~s}_{1}=-1, \mathrm{~s}_{2}=-1.5, \\
& \mathrm{~K}=-2: \\
& \mathrm{G}(\mathrm{x})=\mathrm{r}_{0}+\mathrm{r}_{1}\left(\mathrm{x}-\mathrm{s}_{0}\right)+\mathrm{r}_{2}\left(\mathrm{x}-\mathrm{s}_{0}\right)^{2}+\mathrm{r}_{3}\left(\mathrm{x}-\mathrm{s}_{0}\right)^{3}
\end{aligned}
$$


then

$\mathrm{A}=0.1475, \mathrm{~B}=0.0000, \mathrm{C}=-0.2040, \mathrm{D}=0.0527$

and

$r_{0}=0.5000, r_{1}=1.0000, r_{2}=0.5000, r_{3}=2.0000$

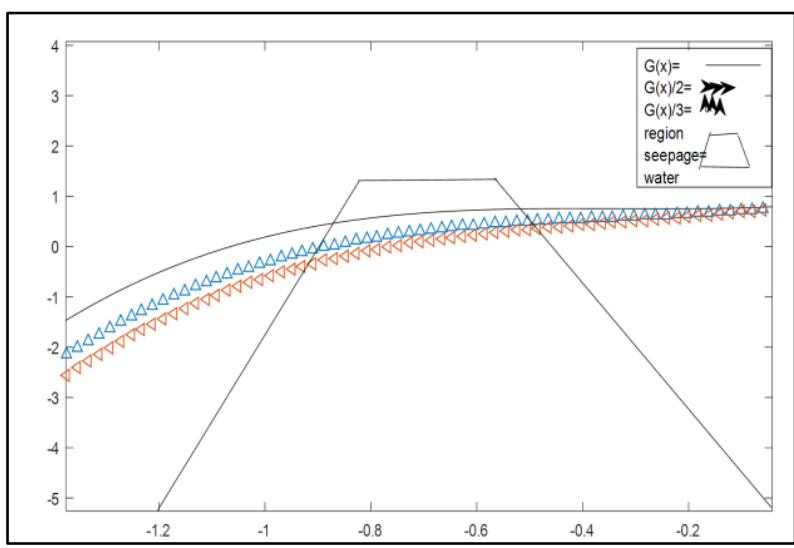

Fig.(4): Approximate free surface of the twodimensional simple.

\section{Conclusions and Recommendations for Future Work:}

From the present work, we may conclude that the variational approach that may be used to formulate and solve many real life problem especially those problems which have so many initial and/or boundary condition and/or those problems which consists boundary condition of free or moving boundaries which must be determined as a part of the solution.

Also, we may recommend some problems for future work concerning to topic of this thesis, such as:

1. Studying the three-dimensional pond seepage problems.

2. Study the physical and mathematical formulation of the invers problem of pond seepage problem.

3. Study and solve the underground water or oil or gas reservoirs.

4. Use other numerical methods to solve the pond seepage problem, such the methods of lines and finite difference methods.

\section{References}

[1] Al-Ani O. H., "Inverse Variational Formulation of the Seepage Problem with Singularity", M.Sc. Thesis, Al-Nahrain University, 2001.

[2] Ali J. A., "On the Mathematical Inverse Problem with Application to Acoustic Wave Scattering". M.Sc. Thesis, Al-
Nahrain University, 1994.

[3] Baiocchi C. and Capelo A., "Variational and Quasi Variational Inequalities: Applications to Free Boundary Problems", John Wiley and Sons Ltd., 1984.

[4] Elliott, C. M., "On a Variational Inequality Formulation of an Electrochemical Machining Moving Boundary Problem and its Approximation by the Finite Element Method", J. Inst. Math. Applics. 25, 121 $131,1980$.

[5] Elsgolic, L. E., "Calculus of Variation", Pergamon Press Ltd., 1962.

[6] Eman, A. H., "Extremum Variational Principles for Boundary Value Problems with Application", M.Sc. Thesis, College of Science, Baghdad University, 1987.

[7] Gelfand I. M. and Fomin S. V., "Calculus of variations", Prentice Hall, Inc., Englewood Cliffs, New Jersey, 1963.

[8] Goodman, T. R., "The Heat Balance and its applications to Problem Involving Change of Phase", Trans., Asme, 80, 335 342, 1985.

[9] Hussain E. A., "Extremum Variational Principles for Boundary Value Problems with Application", M.Sc. Thesis, College of Science, Baghdad University, 1987.

[10] Jabbar S. M., "Variational Approached for Solving Two-Dimensional Free and Moving Boundary Value Problems", M.Sc. Thesis, College of Education, Babylon University, 2001.

[11] Mahlol S. M., "Inverse Problems in Differential Equation with an Application to Localizing Brain Tumors", Ph.D. Thesis, University of Baghdad, 1993.

[12] Magri F., "Variational Formulation for Every Linear Equation”, Int. J. Eng. Sci, 12, 537-549, 1974.

[13] Meyer, G. H., "The Method of Lines and Invariant Imbedding for Elliptic and Parabolic Free Boundary Problems", SIAM J. Numerical Anal. 18, 150 - 164, 1981.

[14] Sahar, M. J., "Variational Approached for Solving Two-Dimensional Free and Moving Boundary Value Problems", M.Sc. Thesis, College of Education, Babylon University, 2000.

[15] Vallentine, H.R., "Applied Hydrodynamics", S.I. Eddition London Butter Worths, 1969. 